\title{
Diurnal variation and reproducibility of predischarge submaximal exercise testing after myocardial infarction
}

\author{
CLIVE E HANDLER, EDGAR SOWTON \\ From the Department of Cardiology, Guy's Hospital, London
}

SUMMARY Diurnal variation and reproducibility of abnormalities occurring during predischarge postinfarction treadmill exercise testing were investigated in 41 patients. Each patient was exercised using a limited Naughton protocol before 0800 and after $1800 \mathrm{~h}$ on two consecutive days. No complications arose. Individual ischaemic abnormalities were poorly reproducible in any patient. No abnormality and no patient showed significant diurnal variation. When the presence of any one of three ischaemic abnormalities (ST segment depression or elevation and angina) was analysed the reproducibility of an ischaemic result in the two morning and the two evening tests was $72 \%$ and $95 \%$ respectively with no significant difference between the two. The reproducibility of an ischaemic result in all four tests was $66 \%$. The reproducibility of the test for either the presence or absence of an ischaemic result was $71 \%$. No training effect could be shown either for the group as a whole or for any individual patient. There were no appreciable differences in either the heart rates or systolic blood pressures during exercise among those patients with non-reproducible ischaemic test results.

Thus it is concluded that an assessment of any one of three ischaemic abnormalities improves the reproducibility of the result of submaximal exercise testing after infarction. Mechanisms other than increased myocardial oxygen consumption related to increased heart rate and systolic blood pressure at submaximal exercise tolerance are needed to explain non-reproducible ischaemic test results.

The result of predischarge submaximal exercise testing after infarction is being used increasingly ${ }^{1}$ in an attempt to identify patients at high risk from subsequent cardiac events and also to select patients for coronary angiography. ${ }^{2}$ Various abnormalities of the test have been correlated with a bad prognosis, ${ }^{3}$ which is associated with the presence of multivessel coronary disease and impaired left ventricular function. ${ }^{4}$ The responses to repeated maximal exercise testing 3-11 weeks after infarction have been studied ${ }^{5} 6$ and have shown an improvement in exercise tolerance with most features of the test, apart from ST segment elevation, ${ }^{7}$ being reproducible. In patients with stable angina but no previous myocardial infarction exercise responses may show diurnal variation. ${ }^{8}$ Because of the important implications of a single positive result for subsequent management we investigated the reproducibility and diurnal variation of abnormalities of

Requests for reprints to Dr C E Handler, Department of Cardiology, Guy's Hospital, London SE1 7RT.

Accepted for publication 10 May 1984 known prognostic value from a predischarge submaximal exercise test.

\section{Patients and methods}

Forty one consecutive patients (six women and 35 men; age range 34-66 (mean 51) years) with uncomplicated acute myocardial infarction were studied. Three patients had had a previous myocardial infarction. All patients fulfilled at least two of the following diagnostic criteria for acute myocardial infarction: $(a)$ retrosternal chest pain consistent with myocardial ischaemia lasting at least 30 minutes, $(b)$ an evolutionary serum enzyme pattern consistent with myocardial necrosis, and (c) electrocardiographic changes.

The diagnostic electrocardiographic changes of transmural myocardial infarction included ST segment elevation, $T$ wave inversion, and the development of $Q$ waves of at least $40 \mathrm{~ms}$ duration and $\geqslant 25 \%$ of the $R$ wave amplitude in the same lead.910 The electrocardiographic criteria for subendocardial myocardial infarction consisted of ST segment depression or $\mathrm{T}$ wave inversion persisting for at least 24 
hours without the development of pathological $Q$ waves. ${ }^{11}$ Thus the sites of infarction were anterior in $18(43 \%)$ patients, inferior in $10(24 \%)$, and posterior in two (5\%), and 11 patients (26\%) had a subendocardial infarction. Patients were excluded from the study if they had the following complications: left ventricular failure clinically or radiographically, hypertension (blood pressure $>165 / 100 \mathrm{~mm} \mathrm{Hg}$ ), and continuing angina or musculoskeletal abnormalities precluding exercise testing. The purpose of the study was explained to the patients, who all agreed to participate. No patient was taking cardiovascular medications at the time of the tests.

\section{EXERCISE TESTING}

Each patient was exercised before 0800 and after $1800 \mathrm{~h}$ (before meals) on two consecutive days between six and 14 days (mean 8) after myocardial infarction. All the tests were performed by the same doctor (CEH). A limited Naughton protocol was followed (Table). A Marquette series 2500 motor driven computerised treadmill was used which was connected to a Mac 1 microcomputer augmented cardiograph and Marquette series 6500 exercise module oscilloscope (Marquette Electronics Inc), which displayed a continuous three channel rhythm strip (leads II, V1, V5) throughout the test. A 12 lead electrocardiogram, heart rate, and blood pressure (cuff sphygmomanometer) were recorded at rest, at the end of each stage of the protocol, at the end of exercise and with the patient sitting, at one minute intervals for five minutes during the recovery period or until the electrocardiogram had returned to the resting appearance. All patients performed the four tests without complications.

The exercise test was stopped when any one of the following end points was reached: angina, a workload of five metabolic equivalents, $70 \%$ of the age predicted maximal heart rate, severe fatigue or dyspnoea, claudication, appearance of frequent $(>10$ per minute) multifocal or paired ventricular extrasystoles, depression of the ST segment $\geqslant 3 \mathrm{~mm}$ as compared with the resting electrocardiogram, or an abnormal blood pressure response, defined as $<10 \mathrm{~mm} \mathrm{Hg}$ increase in systolic pressure during exercise or as a fall in systolic blood pressure occurring during the final workload.

\section{Table Limited Naughton treadmill protocol}

\begin{tabular}{llll}
\hline $\begin{array}{l}\text { Duration } \\
\text { (mins) }\end{array}$ & Mets* & $\begin{array}{l}\text { Speed } \\
(\text { mph })\end{array}$ & $\begin{array}{l}\text { Gradient } \\
(\%)\end{array}$ \\
\hline 2 & 2 & 2 & 0 \\
2 & 3 & 2 & $3 \cdot 5$ \\
2 & 4 & 2 & $7 \cdot 0$ \\
2 & 5 & 2 & 10.5 \\
\hline
\end{tabular}

^ Multiples of resting energy expenditure.
DATA ANALYSIS

Exercise induced abnormalities analysed were: (a) ST segment depression and elevation defined as significant if there was shift on three successive beats of $1 \mathrm{~mm}$ or more for $0.08 \mathrm{~s}$ after the $\mathrm{J}$ point from either the isoelectric ST segment or from the resting level of an ST segment abnormality in any lead, $(b)$ an inadequate blood pressure response, $(c)$ limited exercise duration owing to the attainment of $\geqslant 70 \%$ of the age predicted maximum heart rate, and $(d)$ ventricular arrhythmias.

The reproducibility of the test for ischaemic abnormalities was assessed by comparing the two morning and the two evening tests separately. Diurnal variation was analysed by comparing the two pairs of morning and evening tests.

\section{STATISTICS}

The Yates's corrected $\chi^{2}$ test was used for statistical analysis.

\section{Results}

\section{EXERCISE INDUCED ABNORMALITIES}

None of the exercise induced abnormalities showed significant diurnal variation. There was no significant difference in the reproducibility of any exercise induced abnormality between the two morning and the two evening tests for either the whole group or for any individual patient.

ST segment depression-Fourteen (34\%) patients had exercise induced ST segment depression, and this was reproducible in all four tests in eight (57\%) patients. Five of the six patients with nonreproducible ST depression had this abnormality on one test only (Figure). Five of these six patients and two of the eight patients with reproducible ST segment depression had subendocardial infarctions, but this difference was not statistically significant. Seven of the $14(50 \%)$ patients with ST segment depression had no other ischaemic abnormality.

ST segment elevation-Fourteen (34\%) patients had exercise induced ST segment elevation, and this was reproducible in all four tests in $10(71 \%)$ patients. Although all four patients with non-reproducible ST elevation had this abnormality on the first morning test, none had it on the second morning test, and two and one patient had it on the first and second evening tests respectively. Six of the 14 patients with ST segment elevation had no other ischaemic abnormality.

Angina-Ten (24\%) patients had angina during at least one of the exercise tests, but only four $(40 \%)$ had this abnormality on all four tests. Of the six patients with non-reproducible exercise induced angina, none had this abnormality on the second morning test, two had it on two tests, and four had it on one test only. 

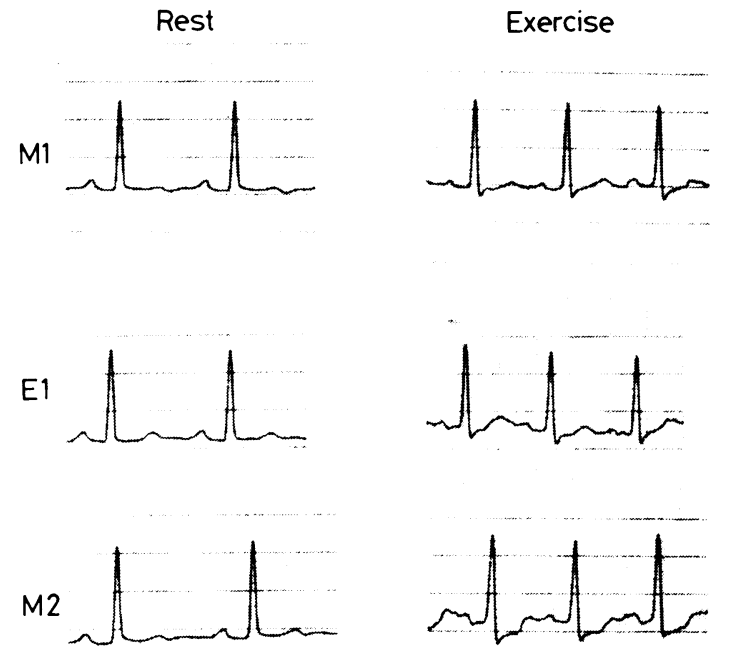

E2
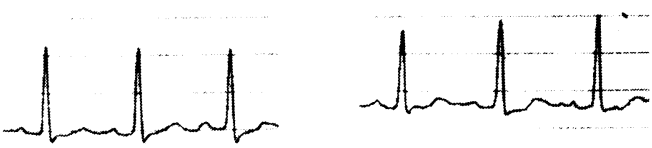

Figure Rest and end exercise electrocardiograms of a patient who developed exercise induced ST segment depression during only one (the third) exercise test. M1, morning (first day); M2, morning (second day); E1, evening (first day); E2, evening (second day).

ST segment elevation and depression-Six (15\%) patients had both ST segment elevation and depression on exercise but only two of these $(33 \%)$ had both exercise abnormalities on all four tests.

$S T$ segment elevation and depression and angina during exercise-Of the 26 patients with one or more of these abnormalities in any one test, only two $(8 \%)$ had all three abnormalities in all four tests.

Inadequate blood pressure response-Five (12\%) patients had this abnormality, but in none was it reproducible in all four tests. One patient had an inadequate response during exercise on three tests.

Limited exercise duration-Six (14\%) patients had limited exercise duration owing to the attainment of $\geqslant 70 \%$ of age predicted maximum heart rate and in four $(66 \%)$ it was totally reproducible. Two $(33 \%)$ patients had this feature on one test only.

Ventricular arrhythmias-Exercise induced ventricular arrhythmias occurred in eight patients, but in no patient were they reproducible in all four tests. They occurred on three tests in one patient and on two tests in another, the remaining six patients having this abnormality on one test only.
REPRODUCIBILITY OF EXERCISE TESTS

For ischaemic abnormalities

The two morning and the two evening tests were analysed separately for reproducibility of ischaemic abnormalities (ST depression, ST elevation, angina). Reproducibility of a test was defined as the presence of one or more of these features in both tests. Of the $26(63 \%)$ patients who had an ischaemic test, $25(96 \%)$ and $22(86 \%)$ had positive tests for reversible myocardial ischaemia in the morning and evening respectively. Of these two groups of patients, 18 of the 25 $(72 \%)$ and 21 of the $22(95 \%)$ had reproducible test results. There was no significant difference between the two morning and the two evening tests regarding the reproducibility of ischaemic abnormalities.

Of the 26 patients with at least one exercise induced ischaemic abnormality present in any one of their four tests, $17(66 \%)$ had four reproducible test results for ischaemia.

For the presence or absence of ischaemic abnormalities Twenty nine of the $41(71 \%)$ patients had totally reproducible test results for either the presence or absence of an ischaemic abnormality.

\section{EFFECT OF REPEATED SUBMAXIMAL EXERCISE TESTING ON HAEMODYNAMIC INDICES}

In the whole group of patients the mean (SD) resting heart rates for the first and fourth exercise tests were $80.2(16.7)$ and $82.7(19.0)$ beats/min respectively. The mean values at end exercise were 119.6 (18.2) and $117.6(18.9)$ beats/min respectively, there being no significant difference between the two pairs of values. Similarly, the mean systolic blood pressures at rest were $114.2(22.6)$ and $115.0(15.8) \mathrm{mm} \mathrm{Hg}$ and at end exercise were $148.7(24.5)$ and $144.9(21.1) \mathrm{mmg} \mathrm{Hg}$, with again no significant difference between the two pairs of values. There were also no significant differences in either end exercise heart rates or systolic blood pressure in the four tests for those patients with non-reproducible exercise induced ischaemic abnormalities.

\section{Discussion}

Several studies have shown that various abnormalities resulting from a predischarge submaximal exercise test after myocardial infarction can stratify patients into high and low risk groups. ${ }^{12-14}$ The rationale for doing the test before hospital discharge is that most of the $10-15 \%$ of the patients who die in the following year do so in the first six weeks ${ }^{15}$ and so their early identification is necessary if appropriate secondary interventions are to be implemented. ${ }^{16} 17$

A predischarge submaximal exercise test is well known to be a safe procedure if the patients are care- 
fully selected and the test supervised by experienced medical staff with full resuscitative facilities available. ${ }^{18}$

The patients in this study were consecutive patients who had had uncomplicated infarcts, and no complications arose from the exercise tests. An end point of five metabolic equivalents was chosen since it approximates to the level of physical activity that such patients are expected to be able to achieve before they leave hospital, although higher workloads have been used safely in symptom limited predischarge tests after infarction. ${ }^{19}$

Exercise induced angina and ST segment depression are well accepted subjective and objective markers of reversible myocardial ischaemia. In patients with previous infarction ST segment elevation occurring during exercise has been found to be similarly associated, ${ }^{20}$ having previously been postulated as merely reflecting localised left ventricular wall dyskinesia. ${ }^{21}$ We therefore included ST segment elevation as one of three features of exercise induced reversible myocardial ischaemia.

Diurnal variation in haemodynamic responses and ST segment displacement during maximal exercise in patients with chronic stable angina has recently been shown by Joy and colleagues. ${ }^{8}$ They suggested that this circadian variation was a consequence of variable vagal parasympathetic tone to the heart. Similarly, studies by Yasue $e t$ al in patients with variant angina have shown diurnal variation in both exercise tolerance and coronary artery tone. ${ }^{22}$

In the present study no exercise induced abnormality showed diurnal variation, and no individual patient showed diurnal variation in subjective or objective features of reversible myocardial ischaemia. Our study differed in two major respects from that reported by Joy et al. ${ }^{8}$ Not only did their patients have chronic stable angina without previous myocardial infarction, but they also used maximal exercise testing.

Our study showed that the reproducibility of individual exercise induced abnormalities considered to be of prognostic value was limited. Starling and coworkers compared the reproducibility of abnormalities resulting from symptom limited treadmill exercise testing performed before discharge and six weeks after myocardial infarction. ${ }^{19}$ They found that ST segment depression was highly reproducible but that angina, ST segment elevation, an inadequate blood pressure response, and ventricular arrhythmias were poorly reproducible. None of our patients was taking cardiovascular drugs at the time of the tests, but $50 \%$ of those studied by Starling et al were. Their patients also had their drug treatment changed between the two tests. They had previously reported ${ }^{23}$ that treatment with digitalis and beta blocking drugs did not affect the prognostic value of ST segment depression. Because digitalis is well known to affect the ST segment, and beta blockers may blunt haemodynamic responses and mask ischaemic abnormalities ${ }^{18}$ it is perhaps surprising that they concluded from their more recent study that the cardiovascular drugs they used did not affect their exercise test results and thus the reproducibility of induced abnormalities.

It is well established that haemodynamic responses alter after repeated maximal exercise in patients both with ${ }^{24}$ and without ${ }^{25}$ ischaemic heart disease. Burkart et al studied predominantly normal subjects and showed an alteration of haemodynamic indicesincluding aortic pressure and heart rate-after repeated testing, ${ }^{25}$ but we could not duplicate this finding. There are several possible reasons for this. Whereas we were exercising recently infarcted hearts to submaximal end points with our tests separated by at least 10 hours, Burkart and colleagues used maximal cycle ergometric exercise, and retested their patients after a 30 minute interval. The results of our own study indicate that repeated submaximal exercise testing after infarction at the intervals used does not give rise to a training effect.

We also found that non-reproducible ST segment depression could not be explained either by differing heart rates-the most reliable and consistent indirect determinant of myocardial oxygen consumption ${ }^{26} 27$ - or by differing systolic blood pressures at end exercise. Thus mechanisms other than increased myocardial oxygen consumption related to heart rate and systolic blood pressure changes are needed to explain non-reproducible objective features of reversible myocardial ischaemia.

That we could not show any diurnal variation in exercise induced abnormalities in this group of patients implies that the test result will not be influenced appreciably if patients are tested either in the morning or evening. In addition, the reproducibility of an ischaemic result was improved by considering three ischaemic abnormalities, and thus the confidence in deciding future clinical management for survivors of recent infarction is accordingly strengthened.

\section{References}

1 Wenger NK, Hellerstein HK, Blackburn H, Castronova SJ. Physician practice in the management of patients with uncomplicated myocardial infarction: changes in the past decade. Circulation 1982; 65: 421-7.

2 Epstein SE, Palmeri ST, Patterson RE. Evaluation of patients after acute myocardial infarction-indications for cardiac catheterization and surgical intervention. $N$ Engl f Med 1982; 307: 1487-92. 
3 Handler CE. Exercise testing early after myocardial infarction: discussion paper. $\mathcal{F} R$ Soc Med 1983; 76: 569-73.

4 Schwartz KM, Turner JD, Sheffield LT, et al. Limited exercise testing soon after myocardial infarction: correlation with early coronary left ventricular angiography. Ann Intern Med 1981; 94: 727-34.

5 Haskell WL, DeBusk R. Cardiovascular responses to repeated treadmill exercise testing soon after myocardial infarction. Circulation 1979; 60: 1247-51.

6 DeBusk RF, Houston N, Haskell W, Fry G, Parker M. Exercise training soon after myocardial infarction. Am $\mathcal{F}$ Cardiol 1979; 44: 1223-9.

7 Starling MR, Crawford MH, Kennedy GT, O'Rourke RA. Treadmill exercise tests predischarge and six weeks post-myocardial infarction to detect abnormalities of known prognostic value. Ann Intern Med 1981; 94: 7217.

8 Joy M, Pollard CM, Nunan TO. Diurnal variation in exercise responses in angina pectoris. $\mathrm{Br}$ Heart $\mathcal{F}$ 1982; 48: $156-60$.

9 Madias JE, Chahine RA, Gorlin R, Blacklow DJ. A comparison of transmural and non-transmural acute myocardial infarction. Circulation 1974; 49: 498-507.

10 Rigu P, Murray M, Taylor DR, Weisfeldt ML, Strauss HW, Pitt B. Hemodynamic and prognostic findings in patients with transmural and non-transmural infarction. Circulation 1975; 51: 1064-70.

11 Szklo M, Goldberg R, Kennedy HL, Tonascia JA. Survival of patients with nontransmural myocardial infarction: a population-based study. Am $\mathcal{F}$ Cardiol 1978; 42: 648-52.

12 Granath A, Södermark T, Winge T, Volpe U, Zetterquist $\mathrm{S}$. Early work load tests for evaluation of long-term prognosis of acute myocardial infarction. Br Heart $\mathcal{F}$ 1976; 39: 758-63.

13 Théroux P, Waters DD, Halphen C, Debaisieux JC, Mizgala HF. Prognostic value of exercise testing soon after myocardial infarction. $N$ Engl $\mathcal{F}$ Med 1979; 301: 341-5.

14 Davidson DM, DeBusk RF. Prognostic value of a single exercise test three weeks after uncomplicated myocardial infarction. Circulation 1980; 61: 236-42.

15 Cole DR, Singian EB, Katz LN. The long-term prognosis following myocardial infarction, and some factors which affect it. Circulation 1954; 9: 321-34.
16 Norwegian Multicenter Study Group. Timolol-induced reduction in mortality and reinfarction in patients surviving acute myocardial infarction. $N$ Engl F Med 1981; 304: 801-7.

17 Wenger NK. Uncomplicated acute myocardial infarction: long-term management. Am $\mathcal{f}$ Cardiol 1983; 52: 658-60.

18 Atterhög J-H, Ekelund L-G, Kaijser L. Electrocardiographic abnormalities during exercise 3 weeks to 18 months after anterior myocardial infarction. Br Heart $\mathcal{F}$ 1971; 33: 871-7.

19 Starling MR, Crawford MH, O'Rourke RA. Superiority of selected treadmill exercise protocols predischarge and six weeks post infarction for detecting ischemic abnormalities. Am Heart $\mathcal{f}$ 1982; 104: 1054-60.

20 Fox KM, Jonathan A, Selwyn A. Significance of exercise-induced ST segment elevation in patients with previous myocardial infarction. Br Heart $\mathcal{F}$ 1983; 49: 15-9.

21 De Feyter PJ, Majid PA, Van Eenige MJ, Wardeh R, Wempe FN, Roos JP. Clinical significance of exerciseinduced ST segment elevation: correlative angiographic study in patients with ischaemic heart disease. Br Heart $\mathcal{F}$ 1981; 46: 84-92.

22 Yasue H, Omote S, Takizawa A, Nagao M, Miwa K, Tanaka S. Circadian variation of exercise capacity in patients with Prinzmetal's variant angina: role of exercise-induced coronary arterial spasm. Circulation 1979; 59: 938-48.

23 Starling MR, Crawford MH, Kennedy GT, O'Rourke RA. Exercise testing early after myocardial infarction: predictive value for subsequent unstable angina and death. Am F Cardiol 1980; 46: 909-14.

24 Malmborg RO. A clinical and hemodynamic analysis of factors limiting the cardiac performance in patients with coronary heart disease. Acta Med Scand 1965; suppl 426: 5-94.

25 Burkart F, Barold S, Sowton E. Hemodynamic effects of repeated exercise. Am $\mathcal{F}$ Cardiol 1967; 20: 509-15.

26 Detry JMR, Piette F, Brasseur LA. Hemodynamic determinants of exercise ST segment depression in coronary patients. Circulation 1970; 42: 593-9.

27 Nelson RR, Gobel FL, Jorgensen CR, Wang K, Wang $\mathrm{Y}$, Taylor HL. Hemodynamic predictors of myocardial oxygen consumption during static and dynamic exercise. Circulation 1974; 50: 1179-89. 